\title{
Synthesis of Equally Excited Linear Arrays
}

\author{
A. KUMAR, MEMBER, IEEE, AND P. K. MURTHY
}

\begin{abstract}
Synthesis of linear arrays employing a perturbation technique to achieve sidelobe reduction by varying element positions alone or phases of antenna currents alone, keeping magnitudes of antenna currents equal, is discussed. Both the mean squared and mini-max error criteria are employed. The technique may also be used to yield patterns with all sidelobes equal.
\end{abstract}

\section{INTRODUCTION}

Synthesis of linear antenna arrays to yield certain prescribed characteristics has been studied to a great depth [1], [2]. A case of practical utility may be the one in which magnitudes of the antenna currents are constrained to be equal, synthesis being carried out by varying only the element positions or phases of the antenna currents. Sidelobe reduction has been effected by Hamngton [3] and Ishimaru [4] by employing unequal spacings and by Harrington [5] and Ma [6] by using nonuniform progressive phase shift. Baklanov et al. [7] have described a method for synthesizing equally excited and unequally spaced arrays having patterns with all sidelobes of equal magnitudes.

Manuscript received May 24, 1976; revised September 16, 1976.

A. Kumar is with the Department of Electrical Communication Engineering, Indian Institute of Science, Bangalore-560012, India.

P. K. Murthy was with the Department of Electrical Communication Engineering, Indian Institute of Science, Bangalore, India. He is now with the Rockets and Missiles Laboratory, Department of Aeronautical Engineering, Indian Institute of Science, Bangalore-560012, India. 


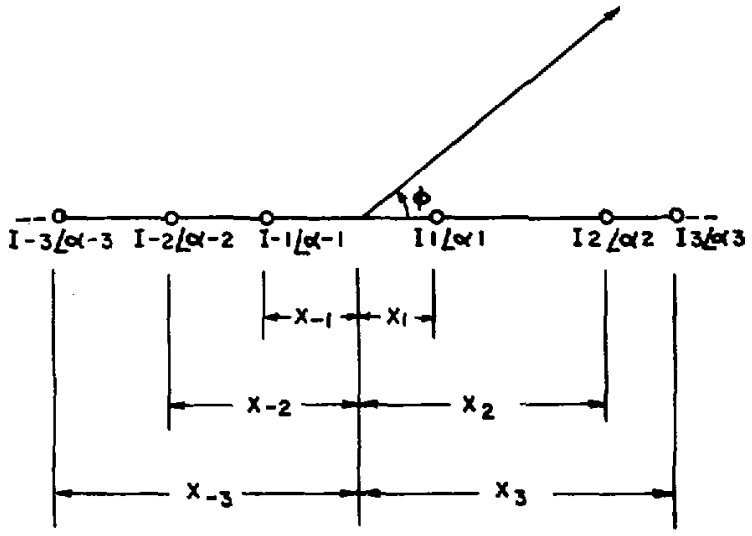

Fig. 1. Geometry of linear array.

\section{THEORY}

Theories of iterative perturbation techniques employed for the synthesis of arrays for the case when only the element positions are varied and also for the case when only the phases of antenna currents are perturbed are given below.

\section{A. Unequally Spaced Arrays}

The array factor of an $N$ (say, even)-element, centersymmetric, equally excited linear array (Fig. 1) at the start of the $(1+1)$ th iteration is given by

$f_{s}^{l}(\bar{X}, \phi)=\frac{2}{x} \sum^{N / 2} \cos \psi_{k}^{l}$, where $\psi_{k}^{l}=\beta x_{k}{ }^{l} \cos \phi+\alpha_{k}^{l}$.

In order to obtain a new set of element positions which yields a closer approximation to the desired pattern, the element positions are perturbed. That is, $x_{k}^{l} \rightarrow x_{k}^{l}+\delta_{k}^{l}$. Correspondingly the array factor changes as given below:

$$
\begin{aligned}
f_{s}^{l} \rightarrow & \frac{2}{N} \sum_{k=1}^{N / 2}\left[\cos \psi_{k}{ }^{l} \cos \left(\beta \delta_{k}{ }^{l} \cos \phi\right)\right. \\
& \left.-\sin \psi_{k}{ }^{l} \sin \left(\beta \delta_{k}^{l} \cos \phi\right)\right] .
\end{aligned}
$$

Assuming $\delta_{k} l$ to be small to the first order of approximation, the expression for the array factor becomes

$$
f_{s}^{l+1}=\frac{2}{N}\left[\sum_{k=1}^{N / 2} \cos \psi_{k}^{l}-\sum_{k=1}^{N / 2} \delta_{k}^{l} V_{k x^{l}}\right]
$$

where

$$
V_{k x^{l}}=\beta \cos \phi \sin \left(\beta x_{k}^{l} \cos \phi+\alpha_{k}^{l}\right) .
$$

The error $\sigma_{p}{ }^{(1+1)}$, employing the $L_{p}$-norm, between the desired pattern $f_{d}$ and the array factor $f_{s}^{(l+1)}$ is given by

$$
\begin{aligned}
o_{p}^{(l+1)} & =\left\|f_{s}^{(l+1)}-f_{d}\right\|_{p} \\
& =\left\|\left(f_{s}^{l}-f_{d}\right)-\frac{2}{N} \sum_{k=1}^{N / 2} \delta_{k}^{l} V_{k x}^{l}\right\|_{p} .
\end{aligned}
$$

The $\delta_{k}{ }^{l}$ are now so chosen that

$$
\frac{2}{N} \sum_{k=1}^{N / 2} \delta_{k}^{l} V_{k x} l
$$

is the best approximation to $\left(f_{s}^{l}-f_{d}\right)$ which is nothing but the error function of the previous iteration. The minimization of $\sigma_{p}{ }^{(l+1)}$ for the cases of $p=2$ and $p=\infty$ is dealt with in $[8]$. The iterative process is carried on until no further reduction in the error becomes possible.

\section{B. Nonuniformly Phased Arrays}

A closer approximation to the desired pattern may also be obtained by perturbing the phases $\alpha_{k}{ }^{l}$ of the antenna currents iteratively. After the lth iteration, let the phases $\alpha_{k}^{l}$ be perturbed as

$$
\alpha_{k}^{l} \rightarrow \alpha_{k}^{l}+\nu_{k}^{l}
$$

Applying (6) to (1) and, as before, assuming the perturbations $\nu_{k}^{l}$ to be small to the first order of approximation, give

$$
f_{s}^{(l+1)}=\frac{2}{N}\left[\sum_{k=1}^{N / 2} \cos \psi_{k}^{l}-\sum_{k=1}^{N / 2} v_{k}^{l} V_{k \alpha}{ }^{l}\right],
$$

where

$$
V_{k \alpha^{l}}=\sin \psi_{k}^{l},
$$

and

$$
\sigma_{p}{ }^{(l+1)}=\left\|\left(f_{s}^{l}-f_{d}\right)-\frac{2}{N} \sum_{k=1}^{N / 2} v_{k}^{l} V_{k \alpha^{l}}\right\|_{p}
$$

Note that the form of (8) is similar to that of (5) and hence $\nu_{k}^{l}$ may be determined in a similar manner.

\section{Patterns with all Sidelobes Equal}

Note that if the desired pattern $f_{d}(\phi)$ is a continuous function having nonzero values over a part of the $\phi$ plane and zero outside it and if this pattern is synthesized employing the $L_{\infty}$-norm, then, in view of the equal ripple property of the mini-max approximation [8], the synthesized pattern may be expected to have sidelobes of equal magnitudes in the regions where $f_{d}(\phi)$ is zero. Hence, arrays having patterns with all sidelobes of equal magnitudes may be synthesized by using the techniques described in the preceding sections.

\section{NUMERICAL EXAMPLES}

Some examples are presented in this section to illustrate the techniques described above. In order to keep the computations simple: the number of elements is chosen to be six.

\section{A. Illustration I}

The following Gaussian pattern is synthesized employing the $L_{2}$-norm by a six-element center-symmetric array:

$$
f_{d}(\phi)=\exp \left[-15\left(\phi-\frac{\pi}{2}\right)^{2}\right]
$$

Since $f_{d}(\phi)$ is a broadside pattern, $\alpha=0$. The weight function is chosen to be constant equal to $2 / \pi$ so that

$$
\int_{0}^{\pi / 2} w(\phi) d \phi=1
$$


TABLE I

SYNTHESIS USING THE $L_{\infty}$-NORM

\begin{tabular}{|c|c|c|c|c|c|c|c|c|c|}
\hline \multirow[b]{2}{*}{ NO. } & \multicolumn{3}{|c|}{$\begin{array}{l}\text { Initial Element } \\
\text { Positions (in } \\
\text { wavelengths) }\end{array}$} & \multicolumn{3}{|c|}{$\begin{array}{l}\text { Final Element } \\
\text { Positions (in } \\
\text { wavelengths) }\end{array}$} & \multirow{2}{*}{$\begin{array}{l}\text { No. of } \\
\text { Iterations }\end{array}$} & \multirow{2}{*}{$\begin{array}{l}\text { Side- } \\
\text { lobe } \\
\text { Level } \\
\text { (dB) }\end{array}$} & \multirow{2}{*}{$\begin{array}{l}\text { Beam- } \\
\text { width } \\
(\infty-d B \\
\text { down })\end{array}$} \\
\hline & $x_{1}^{\prime}$ & $x_{2}^{\prime}$ & $x_{3}^{\prime}$ & $x_{1}$ & $x_{2}$ & $x_{3}$ & & & \\
\hline 1. & 0.25 & 0.75 & 1.25 & & & & 5 & & \\
\hline 2. & 0.2 & 0.6 & 1.0 & 0.252 & 0.503 & 1.122 & 3 & -20.21 & $50.8^{\prime \prime}$ \\
\hline 3. & $\begin{array}{l}0.175 \\
0.15\end{array}$ & 0.525 & 0.875 & & & tion & 4 & & \\
\hline
\end{tabular}

ment positions (expressed in wavelengths):

$$
\text { 1) } x_{1}{ }^{\prime}=0.25, \quad x_{2}{ }^{\prime}=0.75, \text { and } x_{3}{ }^{\prime}=1.25
$$

and

2) $x_{1}{ }^{\prime}=0.15, \quad x_{2}{ }^{\prime}=0.45$, and $x_{3}{ }^{\prime}=0.75$.

In each case, the iterative process has converged to the final element-positions given by $x_{1}=0.260, x_{2}=0.480$, and $x_{3}=$ 1.083. The desired and synthesized patterns are shown in Fig. 2. Note that the sidelobe level has been reduced to -20.4 dB.

The Gaussian pattern given by (9) has also been synthesized employing the $L_{\infty}$-norm making use of Lawson's algorithm [9]. The iteration process is initiated from four different sets of initial element positions. The results are tabulated in Table I. The desired and synthesized patterns are given in Fig. 2. Note that the sidelobes formed in the region where the desired pattern is close to zero, are of equal level (see Section II-C).

\section{B. Illustration 2}

Let the desired pattern coincide with the major lobe of the pattern of a sixelement, $0.2 \lambda$ spaced, Hansen-Woodyard [10] endfire array and let it be zero everywhere else. That is,

$$
f_{d}(\phi)= \begin{cases}\frac{\sin N-}{N} \frac{\psi}{\sin \frac{\psi}{2}}, & -\frac{2 N}{2 N} \leqslant \psi<\frac{}{N-1} \\ 0, & \left.-2 \beta d-\frac{\pi}{N-1}<1\right)<-\frac{57}{2 N}\end{cases}
$$

where $\psi=\beta d \cos \phi-\beta d-\pi /(N-1)$.

Let a sixelement, $0.2 \lambda$ spaced array be synthesized by iteratively perturbing the phases of the antenna currents until the best match is obtained employing the $L_{2}$-norm. Starting from the initial values of phases given by.

$$
\alpha_{k}{ }^{1}=-\alpha_{k}{ }^{1}=(k-1 / 2)(-\beta d-)
$$

the iteration process has converged to the following final values (in radians) in only four iterations:

$$
\alpha_{1}=-\alpha_{-1}=-0.894, \quad \alpha_{2}=-\alpha_{-2}=-2.582,
$$

and

$$
\alpha_{3}=-\alpha_{-3}=-4.866
$$

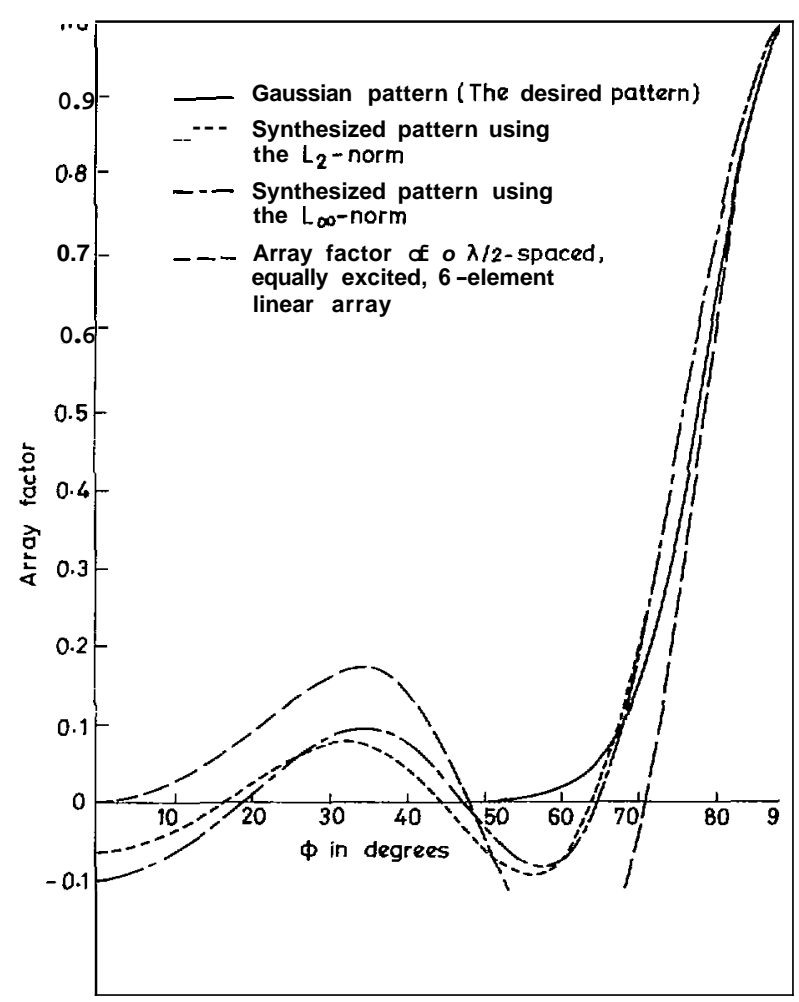

Fig. 2. Synthesis of Gaussian patterns by unequally spaced equally excited linear array.

The desired and the synthesized patterns are shown in Fig. 3. Note that the sidelobe level is decreased from $-6.6 \mathrm{~dB}$ to $-11.3 \mathrm{~dB}$. However, the main beam radiation efficiency [11], which is a measure of the super-directivity, increases only slightly from 26.3 to 27.0 percent.

The pattern has also been synthesized employing the $L_{\infty^{-}}$ norm. In this case the iteration process converges to the following final values (in radians) in only three iterations:

$$
\begin{aligned}
& \alpha_{1}=-\alpha_{-1}=-0.914, \quad \alpha_{2}=-\alpha_{-2}=-2.450, \\
& \alpha_{3}=-\alpha_{-3}=-4.733 .
\end{aligned}
$$

The synthesized pattern is shown in Fig. 3. Note that a considerable reduction in sidelobe level is effected though there is an increase in the beamwidth $\left(56.8^{\circ}\right)$. Further, the main beam radiation efficiency is increased from 26.4 to 35.3 percent thus making the array less super-directive. The directivity of the synthesized array is 11.8 and is, therefore, significantly higher than that of the Hansen-Woodyard array which is only 8.2. That is, there is a simultaneous increase in both the main beam radiation efficiency and the directivity. 


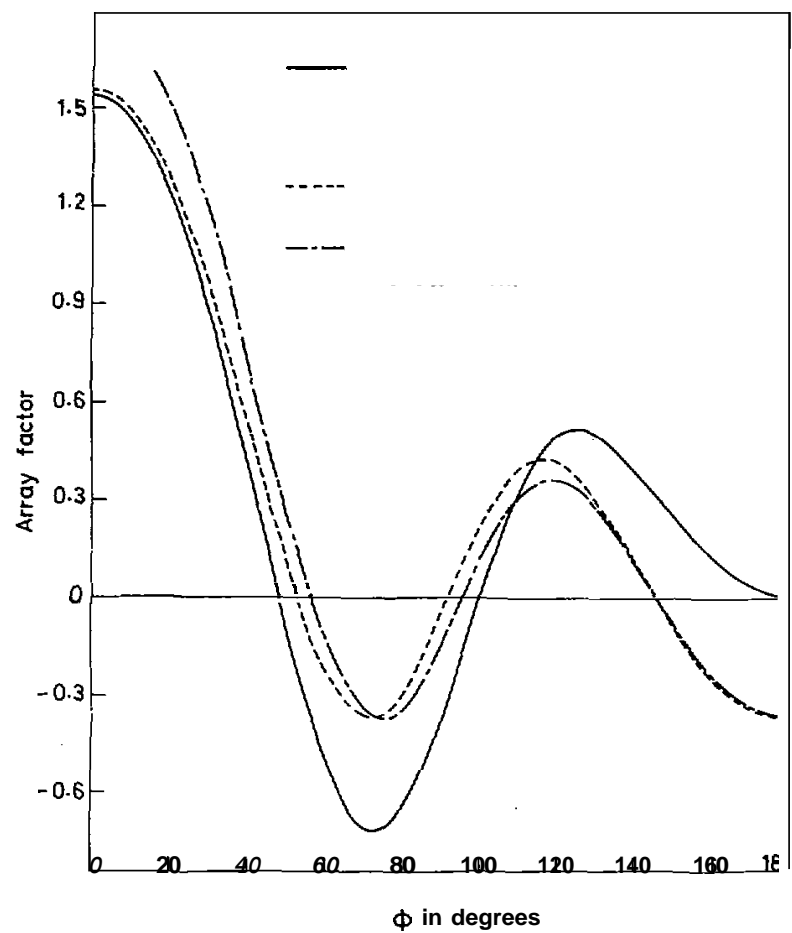

Fig. 3. Synthesis of Hansen-Woodyard pattern by nonuniformly phased equally excited linear array.

Further, note that the sidelobes are of equal magnitudes (see Section II-C).

\section{CONCLUDING REMARKS}

From the theory and illustrations presented in this communication, it is clear that a considerable reduction in the sidelobe level may be obtained by allowing the interelement spacings or phases of antenna currents to vary. Note that the techniques do not involve any trial and error procedures. The convergence of the interation to the final solution has been found to be fast, usually within ten iterations. However, when the $L_{\infty}$-norm is employed, the iteration process may not converge, if the element positions obtained at any stage of the iteration are such that $V_{k}$ do not constitute a Chebyshev set [8]. Ma and Walters [12] point out that this condition may occur if the average interelement spacing is greater than $\lambda / 2$. Hence techniques described here are applicable to arrays with small spacings.

Note that reduction of the sidelobe level is not because of any tradeoff relationship with beamwidth, directivity, or efficiency, but because more parameters are under the control of the designer. For instance, in illustration 2, the reduction in the sidelobe level is accompanied by an increase in the directivity and also in the main beam radiation efficiency.

The technique, described here, for the synthesis of optimum arrays is considerably simpler than that employed by Baklanov et al. [7]. However, it may be noted that this method ensures equality of levels of only those sidelobes that are formed in the region where the desired pattern is zero.

\section{REFERENCES}

[1] R. E. Collin and F. J. Zucker, Antenna Theory. New York: McGraw-Hill, 1969, Part I, Chapters 5-7.
[2] P. K. Murthy, "Synthesis of Antenna Arrays," Ph.D. dissertation, Department of Electrical Communication Engineering, Indian Institute of Science, Bangalore-560012, September 1974.

[3] R. F. Harrington, "Sidelobe Reduction by Non-uniform Element Spacing," IRE Trans. Antennas Propagat., Vol. AP-9, pp. 187192, March 1961.

[4] A. Ishimaru. "Theory of Unequally Spaced Arrays," IRE Trans. Antennas Propagat., Vol. AP-10, pp. 691-702, November 1962.

[5] R. F. Harrington, "Modified Progressive Phasing for End Fire Arrays," IEEE Trans. Antennas Propagat., Vol. AP-11, pp. 1317, January 1963.

[6] M. T. Ma, "Linear arrays with Non-uniform Progressive Phase Shift,” IEEE Int. Conv. Rec., Part-1, pp. 70-76, March 1963.

[7] Ye. V. Baklanov, V. L. Pokrovskiy, and G. I. Surdutovitch, "A Theory of Linear Antenna with Unequal Spacing," Radio Engineering and Electronic Physics, Vol. 7, pp. 905-913, 1962.

[8] J. R. Rice, The Approximation of Functions, Vol. 1. New York: Addison-Wesley, 1964.

[9] P. K. Murthy and A. Kumar, "Synthesis of Linear Antenna Arrays," IEEE Trans. Antennas Propagat., Vol. AP-24, pp. 865-870, November 1976.

[10] W. W. Hansen and J. R. Woodyard, "A New Principle in Directional Antenna Design," Proc. IRE, Vol. 26, pp. 333-345, March 1963.

[11] D. K. Cheng, "Optimization Techniques for Antenna Arrays," Proc. IEEE, Vol. 59, No. 12, pp. 1664-1674, December 1971.

[12] M. T. Ma and L. C. Walters, "Synthesis of Non-uniformly spaced Antenna Arrays Yielding Approximately equal Sidelobes," Radio Sci. 1(New series), vol. 1,pp. 601-608, May 1966.

\section{A Coupled Resonant Directive Antenna Consisting of a Dipole, a Reflector, and Disks}

\author{
NOZOMU HASEBE, MEMBER IEEE, \\ AND TOMOYUKI ZAMA
}

Abstract-An experimental study of a coupled resonant directive antenna consisting of a $\lambda / 2$ dipole ( $h$ is wavelength), a reflector of about $2 \lambda$ diameter, and disks of about $\lambda / 2$ diameter is presented. The $\lambda / 2$ spacing between the reflector and a disk and the $\lambda / 2$ spacing between disks constitute leaky resonant cavities which are mutually coupled. The antenna shows a relatively high gain per element and a good suppression of sidelobes. Its simple and axially symmetric structure makes the antenna suitable for elements of an array antenna. Characteristics are mostly studied by models at $5 \mathrm{GHz}$ and summarized in the form of equicontour maps. The antenna at $1.6 \mathrm{GHz}$ yields a gain of $12.8 \mathrm{~dB}$ above a $\lambda / 2$ dipole. An array of 16 elements at $400 \mathrm{MHz}$ yields a gain of $24.3 \mathrm{~dB}$ above an isotropic source.

\section{INTRODUCTION}

An antenna consisting of a dipole, a reflector, and disks has been a subject of many studies. Ehrenspeck studied an antenna with one disk spaced $\lambda / 2$ from the reflector [1]-[3], which was called a short-backfire antenna. He also showed that a rim surrounding the reflector improved the gain and the sidelobe suppression [4]. Hass showed that the use of two disks yields comparable characteristics without using rim [5]. An antenna

Manuscript received January 19, 1976; revised July 20, 1976.

The authors are with the Institute of Industrial Science, University of Tokyo, Roppongi, Tokyo, Japan. 